The following paper posted here is not the official IEEE published version. The final published version of this paper can be found in the Proceedings of the IEEE Annual Conference on Industrial Electronics (32nd : 2006 : Paris, France):pp. 958-963

Copyright @ 2006 IEEE.

Personal use of this material is permitted. However, permission to reprint/republish this material for advertising or promotional purposes or for creating new collective works for resale or redistribution to servers or lists, or to reuse any copyrighted component of this work in other works must be obtained from the IEEE. 


\title{
Investigation of Static Eccentricity Fault Frequencies using Multiple Sensors in Induction Motors and Effects of Loading
}

\author{
Randy Supangat ${ }^{1}$, Jason Grieger, Nesimi Ertugrul, Wen L. Soong, Douglas A. Gray, and Colin Hansen* \\ School of Electrical and Electronic Engineering and ${ }^{*}$ School of Mechanical Engineering \\ University of Adelaide \\ Australia \\ ${ }^{1}$ rsupang@eleceng.adelaide.edu.au
}

\begin{abstract}
A significant number of studies have been reported in the literature on the detection of eccentricity faults in induction machines; however these have generally used one type of sensor such as current or vibration, and not studied in detail the effect of loading. This paper examines the use of fault frequency components in the current, flux and vibration sensor signals with the aim of both detecting and estimating the severity of static eccentricity faults in the presence of load variations. The research is based on an extensive series of sensor measurements taken using a specially modified $2.2 \mathrm{~kW}$ induction machine under varying conditions of static eccentricity and loading.
\end{abstract}

\section{INTRODUCTION}

Eccentricity faults are a common type of fault in induction motors and account for about $12 \%$ of the total number of induction motor failures [1]. They can be caused by installation and handling errors, manufacturing defects and wear. In addition, such faults can potentially induce other faults, such as misalignment, bearing damage and lamination damage, and may eventually cause catastrophic failure.

There are two types of eccentricity fault associated with induction motors, namely static eccentricity (SE) and dynamic eccentricity (DE). Static eccentricity is where the air gap difference between the stator and the rotor varies but the position of the minimum air gap is fixed, while dynamic eccentricity is where the position of the minimum air gap rotates with the rotor.

There has been significant research work into condition monitoring techniques for detecting static and dynamic eccentricity faults in induction machines using characteristic fault frequencies in the current and vibration signals [2-6].

In [2], a $11 \mathrm{~kW}$ machine was studied to detect and distinguish different levels of severity of both static ( $\mathrm{SE}=0$ to $50 \%$ in 6 equal steps) and dynamic eccentricity ( $\mathrm{DE}=0 \%$, $20 \%, 40 \%$ ) using the fault frequency components (Eqn. 2 in Table 1) in the current spectrum, under 3 different loading conditions $(0 \%, 50 \%$ and $100 \%)$. It was demonstrated in the paper that the sideband current increases as the severity of the faults increases, and the rotor frequency component was used to determine the type of the eccentricity fault (static or dynamic). It was also reported that the sideband current variation for static eccentricity, in the absence of dynamic eccentricity, is relatively small and hence hard to detect.

The work in [6] described an eccentricity investigation for a $1.45 \mathrm{MW}$ industrial machine. It was reported that it was possible to distinguish a "faulty" and "healthy" motor by monitoring the fault frequency components (Eqns. 1 and 2 in Table 1) in the current spectrum under one particular load.

A simulated $3 \mathrm{hp}$ machine (using the modified winding function approach) was reported in [4]. The different levels of both static and dynamic eccentricity using (Eqns. 1 and 2) in the current spectrum, were given in [4], which only considered two different loading conditions (no-load and load). In the study, the simulation results were verified using a practical motor of identical ratings. It was concluded that the fault frequency components given by (Eqn. 2) are present in both the healthy and faulty motors and the difference between them is marginal when no dynamic eccentricity is introduced (e.g. $\mathrm{SE}=40 \%$ and $\mathrm{DE}=0 \%$ ). However, the magnitude difference between the healthy and faulty motors becomes more evident when dynamic eccentricity is introduced to the motor (e.g. $\mathrm{SE}=40 \%$ and $\mathrm{DE}=40 \%$ ).

In summary, the majority of the previous work has focussed on: the combined effects of both static and dynamic eccentricity; examined only a few eccentricity fault frequencies in one of the sensor signal types; and concentrated on large motors. However, it is critical to know what the most relevant fault frequencies for detecting static and dynamic eccentricity are and how the load variation affects the fault detection.

This paper examines the use of fault frequency components in the current, flux and vibration sensor signals to try to both detect and estimate the severity of static eccentricity faults in the presence of load variations. The research is based on an extensive series of sensor measurements taken using a specially modified $2.2 \mathrm{~kW}$ induction machine under varying conditions of static eccentricity and loading.

\section{CHARACTERISTIC ECCENTRICITY FAULT FREQUENCY EQUATIONS}

As is well known, the induction motor is a highly symmetrical machine, whose symmetry is distorted by the presence of a fault. The eccentricity related distortion in induction motors produce characteristic fault frequencies in the stator current, axial flux and vibration as given in the Eqns. (1-8) in Table 1.

The table below shows the earlier research studies [2-6] that have utilised these eccentricity fault frequency equations. The previous studies primarily utilised only one of these equations and a single sensor type, and found that a significant increase in the magnitude of a characteristic fault frequency can indicate an eccentricity fault. However, neither the relationship between the degree of eccentricity and the magnitude of the fault frequency component nor the sensitivity of these fault frequencies to changes in loading and misalignment has been reported in detail in the literature. 
TABLE I

ECCENTRICITY FAULT FREQUENCY EQUATIONS

\begin{tabular}{|c|c|c|c|c|}
\hline Eccentricity Fault Frequencies & $\begin{array}{l}\text { Sensor } \\
\text { Types }\end{array}$ & $\begin{array}{c}\text { Eccentricity } \\
\text { Types }\end{array}$ & $\begin{array}{l}\text { Refer } \\
\text { ences }\end{array}$ & $\begin{array}{l}\text { Eq. } \\
\text { No. }\end{array}$ \\
\hline$D E S E 1=f\left[\left(k R \pm n_{d}\right) \frac{(1-s)}{p} \pm v\right.$ & $\begin{array}{c}\text { Current, } \\
\text { Flux }\end{array}$ & $\begin{array}{l}\text { Dynamic, } \\
\text { Static }\end{array}$ & $\begin{array}{l}{[3,4,5} \\
, 6]\end{array}$ & (1) \\
\hline$D E S E 2=f \pm f_{r}$ & $\begin{array}{c}\text { Current, } \\
\text { Flux }\end{array}$ & $\begin{array}{c}\text { Dynamic, } \\
\text { Static }\end{array}$ & $\begin{array}{l}{[2,3,4} \\
, 5,6]\end{array}$ & (2) \\
\hline$D E S E 3=2 f \pm f_{r}$ & Vibration & $\begin{array}{c}\text { Dynamic, } \\
\text { Static }\end{array}$ & [2] & (3) \\
\hline 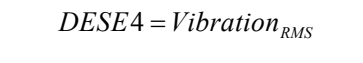 & Vibration & $\begin{array}{l}\text { Dynamic, } \\
\text { Static }\end{array}$ & & (4) \\
\hline$D E 1=f_{r}$ & Vibration & Dynamic & [2] & (5) \\
\hline$D E 2=2\left(f \pm f_{r}\right)$ & Vibration & Dynamic & [2] & (6) \\
\hline$S E 1=3 f_{r}$ & Vibration & Static & & (7) \\
\hline$S E 2=2 f$ & Vibration & Static & [2] & (8) \\
\hline
\end{tabular}

where $k$ is any integer, $R$ is the number of rotor bars, $n_{d}=0$ (static eccentricity) and $n_{d}=1,2,3 \ldots$ (dynamic eccentricity), $s$ is the slip, $p$ is the number of pole pairs, $v$ is the order of the stator time harmonics, $f$ is the fundamental supply frequency, and $f_{r}$ is the frequency corresponding to the rotor mechanical speed.

\section{A. Current and Flux Monitoring}

Stator current and axial leakage flux measurements are commonly used for eccentricity fault detection. Eccentricity faults disturb the symmetry of an induction motor, which results in an unbalanced magnetic pull (UMP) and produces known characteristics fault frequencies in the current and flux spectra.

As given in Table 1, Eqn. (1) can be used to detect static eccentricity when $n_{d}$ is set to 0 . Hence the equation can be reduced to the combination of rotor frequency $\left(f_{r}\right)$ and fundamental frequency $(f)$, as given below in (9), which shows that static eccentricity faults give rise to the rotor slotting effects on the induced stator EMF.

$$
D E S E 1=f\left[\left(k R \pm n_{d}\right) \frac{(1-s)}{p} \pm v\right]=k R f_{r} \pm v f
$$

It has been reported in $[2,4]$ that the presence of static eccentricity may also produce dynamic eccentricity components. Therefore, monitoring both the dynamic and static eccentricity fault frequencies even in the presence of only one type of eccentricity faults may be beneficial. Dynamic eccentricity can also be detected using (1) by setting $n_{d}$ to $1,2,3 \ldots$

A limitation of the characteristic eccentricity fault frequencies in (1) is that these eccentricity components may not be present in all types of motors. It was shown by [3] that eccentricity fault detection using (1) is possible only if the number of rotor bars in the motors is given by:

$$
\begin{aligned}
& R=2 p[3(m \pm q) \pm r], \text { or } \\
& R=2 p[3(m \pm q) \pm r] \pm s,
\end{aligned}
$$

where $r=0$ or $1, s=1$ or $2, m \pm q=0,1,2,3, \ldots$

Although (10) is valid for most motors, (11) is valid for motors generating principal slot harmonics (PSH). The difference is because the additional pole pair rotor MMF harmonics, which are produced by the MMF acting on the permeance between the stator and the rotor, are different between the two types of motors [4].

In this study, only the strongest sideband (i.e. the first sideband) of (1) was investigated. Therefore, the investigation considers $k=1, n_{d}=0, v= \pm 1$ when detecting static eccentricity faults, and $k=1, n_{d}=1, v= \pm 1$ when detecting dynamic eccentricity faults.

Equation (2) is also commonly utilised for eccentricity fault frequency components in the current and flux spectra, as they are very strong and hence easy to detect. In addition, unlike (1), the machine constructional information is not required in (2). However, the fault frequency components in (2) are affected by the presence of both static and dynamic eccentricity [2,6]. Therefore, while Eqn. (2) cannot distinguish the type of eccentricity, it is considered reliable in detecting the general level of eccentricity.

\section{B. Vibration Monitoring}

Vibration monitoring is also considered for eccentricity detection as it is non-invasive and can be easily installed on most motors.

As listed in Table 1, the characteristic eccentricity fault frequency components can be found from Eqns. (3,5,6 and 8). Although a detailed derivation of the origin of these fault frequency components are given in [2], there is a lack of detailed investigation and experimentation in the literature using these components.

In this paper, the effect of eccentricity on the vibration RMS level given in (4) is investigated. This is because an eccentricity fault would be expected to increase the overall vibration level in the machine. Note that an increase in vibration level can also be due to many other factors such as imbalance, misalignment, load faults and supply faults (such as unbalanced supply). Furthermore, since eccentricity and misalignment faults are related to the harmonic components of the rotor frequency, $f_{r}$ [2], the first (Eqn. (5)), and third (Eqn. (7)) harmonic of the rotor frequency are also considered in the investigation.

\section{EXPERIMENTAL SETUP AND RESULTS}

In the experimental study, a custom built motor test rig and a high-end data-acquisition system (2 x DAQPAD-6052E boards, 12-bit, from National Instruments) capable of simultaneous sampling at $5 \mathrm{MHz}$ have been used.

Figure 1 shows a sectioned view of the test motor $(2.2 \mathrm{~kW}$, $1415 \mathrm{rpm}, 415 \mathrm{~V}, 4.9 \mathrm{~A}, 4$ poles, 32 rotor bars, Y-connected) with the adjustable static eccentricity facility. The test arrangement was designed to allow the introduction of repeatable and reliable static eccentricity. The motor is loaded by a separately-excited DC generator that is directly coupled to the test motor. As illustrated in Fig. 1, the test motor consists of custom-built motor end-plates which contain an adjustable bearing housing. The graduated screws in the setup allow the operator to introduce static eccentricity with a resolution of $0.01 \mathrm{~mm}$ (nominal airgap is $0.39 \mathrm{~mm}$ ).

In order to ensure the accuracy and the repeatability of the data obtained and to ensure that no other faults (such as 
misalignment) are present during tests, a precision laser alignment tool (Optalign Plus from Prüftechnik) and a torque wrench (Norbar, set at $35 \mathrm{Nm}$ ) were used.

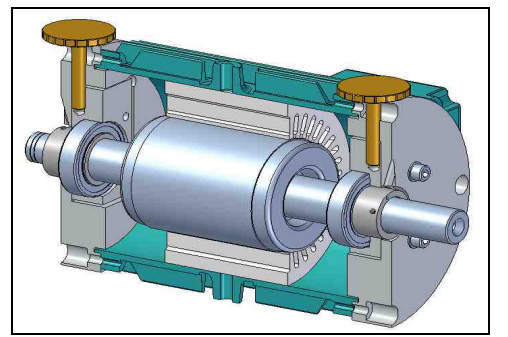

Fig. 1. Sectioned view of the test motor showing the adjustable static eccentricity arrangement.

During the tests, two current sensors, one external axial flux search coil, and two vibration sensors (drive-end horizontal and non-drive end horizontal) were attached to the motor. The five sensor signals were acquired simultaneously using the data acquisition system via an 8th order Butterworth anti-aliasing filter which can be set at either 100 $\mathrm{Hz}$ or $2 \mathrm{kHz}$.

Static eccentricity tests were performed from $-50 \%$ (of the total air gap of the test motor) to $+50 \%$ in nine equal steps (that is, from $-0.2 \mathrm{~mm}$ to $+0.2 \mathrm{~mm}$ ). At each eccentricity level, the load was varied from $2.5 \%$ to $100 \%$ of rated load in ten steps. The load is introduced by connecting resistors in parallel with the DC machine armature and it is estimated by measuring the rotor frequency in the vibration sensor. For each combination of eccentricity and loading level, the sensor signals were measured at two sampling rates:

- $\quad 400 \mathrm{~Hz}$ sampling frequency and 100 seconds sampling time (low-frequency measurement), which allows a very high-resolution frequency analysis $(0.01 \mathrm{~Hz}$ resolution),

- $\quad 8000 \mathrm{~Hz}$ sampling frequency with a sampling time of 5 seconds (high-frequency measurement).
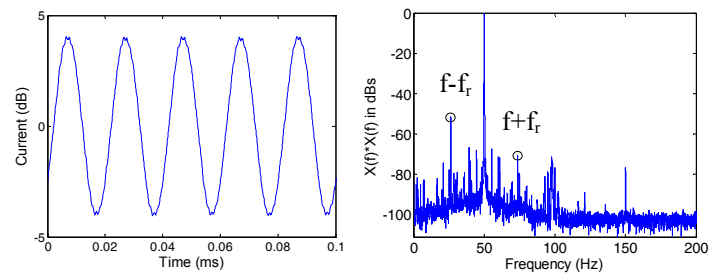

Fig. 2. Typical measured stator current signal (left) and corresponding frequency spectrum (right).

A sample sensor signal (a line current) and corresponding frequency spectrum are given in Fig. 2. The two characteristic fault frequencies given by (2) are also shown in this figure. Note that the frequency components in the current and flux signals are normalised to the fundamental, while the frequency components in the vibration is not normalised.

The results analysed in the paper were obtained under different loading conditions and multiple static eccentricity settings. However, due to the space constraints, only the upper sidebands of the frequency components in Eqns. (2, 3 and 6) and a few components in (1) from a single sensor type have been plotted.

Figure 3 presents the results of the variation of the magnitude of the eccentricity fault frequency components under different loading and eccentricity settings using Eqn. (1). Identical legends are used in the following figures (Figs. 4 to 12) to display the discrete loading conditions and static eccentricity levels. Note that the eccentricity levels in the figures are defined with respect to the ideal "centred" position of the rotor (zero eccentricity), and that negative values of eccentricity indicate that the bearing housings in the test setup are moved down.

Figure 3a demonstrates the dynamic eccentricity fault frequency component $\left(n_{d}=1\right)$ as a function of load and eccentricity. It shows that starting from zero load, the magnitudes of the fault frequency component tend to decrease at light load (0-20\%) but is constant at higher loads. Although a magnitude variation between the different levels of eccentricity can be observed in the plots (Fig. 3a, right), they are not consistent across the different loading conditions. Ideally, the magnitude should increase as the severity of the fault increases as shown in Fig. 3a (right) at higher loads but not low loads.

Similarly, Fig. $3 \mathrm{~b}$ displays the results of (1) when $n_{d}$ is set to 0 (static eccentricity fault frequency component). Although this component is commonly investigated in the current signal, Fig. $3 \mathrm{~b}$ is given for the axial flux signal. The results demonstrate that starting at zero load, the magnitude tends to increase at low loads $(0-30 \%)$ and is constant at higher loads. In addition, as also shown in Fig. 3b (right), the magnitude variation of the studied fault frequency component is quite consistent under different loading conditions. Therefore, it can be concluded that this fault frequency component is a good feature for detecting eccentricity albeit the variation between the different levels of eccentricity is not great (only $\pm 5 \mathrm{~dB}$ ).
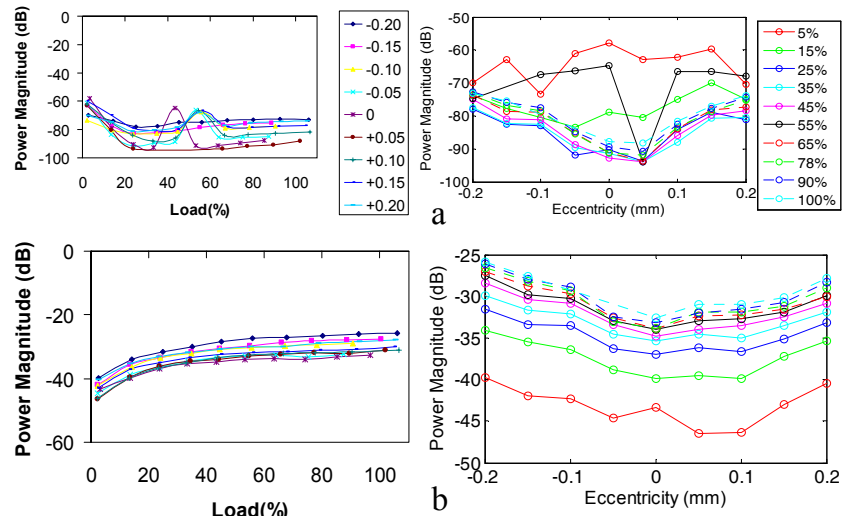

Fig. 3. Magnitude of the peaks as a function of load (left column) and degree of eccentricity (right column) from Eqn. (1) with:

a) current signal with $n_{d}=1, v=1$; b) flux signal with $n_{d}=0, v=1$.

Although the fault frequency component, $f \pm f_{r}$, in (2) is a commonly used feature for detecting eccentricity using the current signal, Fig. 4 presents the test results for the axial flux signals. It was observed that the magnitude of this component is quite strong and steady regardless of the loading conditions (Fig. 4 left). Hence it can be detected easily and reliably.

The results indicate that the magnitude of the healthy 
motor (zero eccentricity) is significantly lower than the magnitude of the faulty motors. However, the magnitude variation between the different levels of eccentricity is found to be relative small and random (Fig. 4 right). The results demonstrate that this feature is able to distinguish healthy and faulty motors relatively easily but is not very effective in separating the different levels of eccentricity.
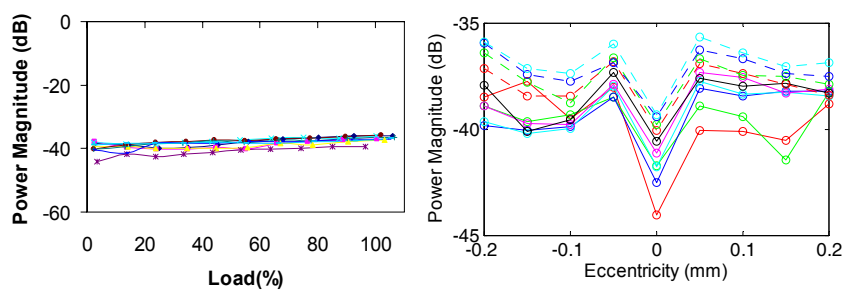

Fig. 4. Magnitude of the component, detected by (2) in the flux signal, as a function of load (left) and degree of eccentricity (right).

Figure 5 displays the experimental results that utilised (3). This fault frequency component is detected from the vibration signal and is not as commonly used as the previous two equations ( 1 and 2 ). The component is generally found to be not very strong $(\sim-90 \mathrm{~dB})$, and may be masked by other stronger components. In addition, the results indicate that the magnitude variation among the different levels of eccentricity is not clear (Fig. 5, right). Hence this component was not found to be a reliable feature for eccentricity detection in the test motor.
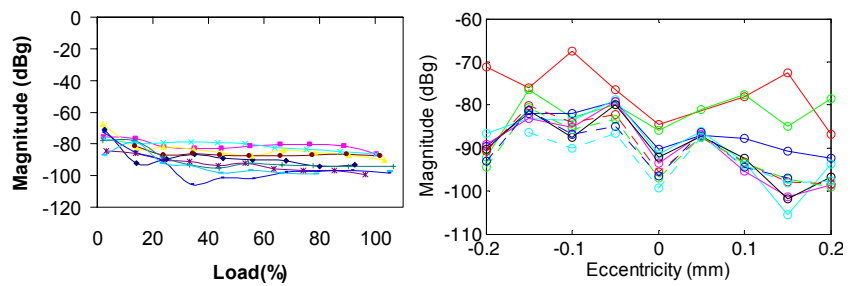

Fig. 5. Magnitude of the component, detected by (3) in the vibration signal, as a function of load (left) and degree of eccentricity (right).

Figure 6 shows the experimental results of vibration RMS under different eccentricity levels and loading conditions. As can be observed in the figure, the vibration RMS level tends to decrease at light load $(0-20 \%)$ then increase slightly at higher loads (Fig. 6, left) as the higher torque in the motor generates more vibration. In the results, the magnitude variation among the different levels of eccentricity is clearly observable (Fig. 6, right), where the vibration level increases as the severity of the fault increases.
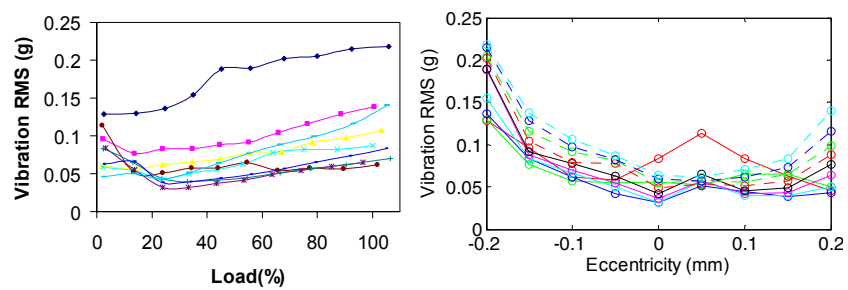

Fig. 6. Vibration RMS using (4) as a function of load (left) and degree of eccentricity (right).

The variation of the vibration RMS levels was found not to be symmetrical around the zero eccentricity position. The figure indicates that more vibration was found when the motor bearing is moved down (-) than when it is moved up $(+)$. A possible explanation is that the original rotor position may not be perfectly centred or the magnetic and flux field distribution of the original rotor position may not be perfectly symmetrical, as would be expected in practical motors.

As given by (5), the rotor frequency component, $f_{r}$, is a commonly used feature for eccentricity and misalignment detection in the vibration spectrum. Figure 7 (left) shows that the magnitude of this component tends to increase at light load $(0-15 \%)$ then level off at higher loads. This component is relatively strong and easy to detect, which is particularly useful to obtain an accurate measurement of the running speed of the motor (and hence slip) even at a very light loads ( $3 \%$ load) as shown in Fig. 7 (left). However, the magnitude variation among the different levels of eccentricity is very small and so is hard to distinguish (Fig. 7 right). Therefore, this component is not suitable for eccentricity detection.
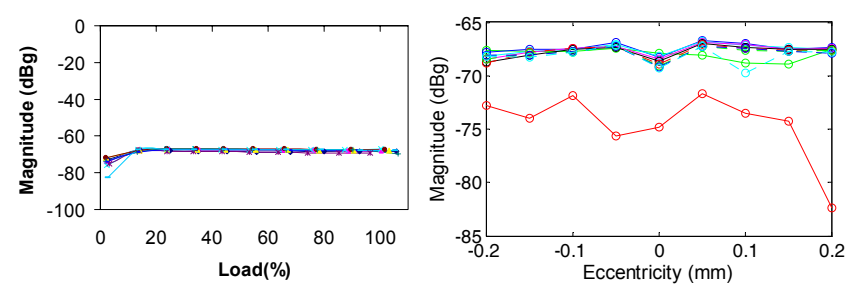

Fig. 7. Magnitude of the component, detected by (5) in the vibration signal, as a function of load (left) and degree of eccentricity (right).

Figure 8 shows the results of the fault frequency component detected by (6) in the vibration spectrum. This fault frequency is one of the less commonly investigated components. The results demonstrate that the magnitude of the fault frequency component tends to decrease at low loads $(0-20 \%)$ then level off at higher loads (Fig. 8, left). The figure also shows that the magnitude is very weak $(\sim-100$ $\mathrm{dB}$ ) and hence may be difficult to detect. The magnitude variation among the different levels of eccentricity is also poor (Fig. 8, right). Therefore, this component may not be a suitable feature for detecting eccentricity faults.
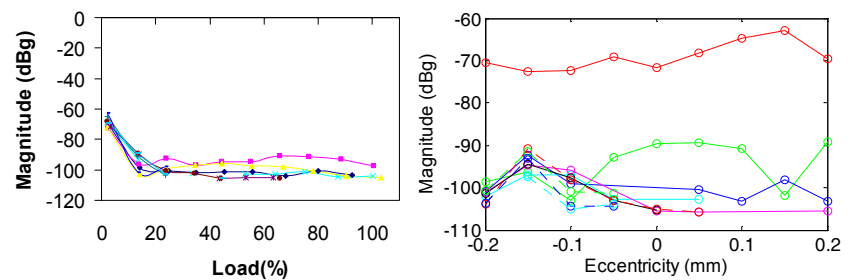

Fig. 8. Magnitude of the component, detected by (6) in the vibration signal, as a function of load (left) and degree of eccentricity (right).

The experimental results given in Fig. 9 using (7) indicate the third harmonic component of the rotor frequency, $3 f_{r}$. The magnitude of this component also tends to decrease at low loads $(0-20 \%)$ then level off at higher loads (Fig. 9 left). In this component, since the magnitude variation among the different levels of eccentricity is generally poor it is not recommended as a feature for eccentricity detection.

Figure 10 presents the experimental results obtained by using (8) in the vibration spectrum. As can be seen in the 
figure (left), the magnitude of this component is relatively steady across the different loading conditions. Although the component is relatively strong and so easy to be detect, the magnitude variation among the different levels of eccentricity is not ideal and unsystematic. The component tends to decrease as the eccentricity is increased in the positive direction (rotor up) but it remains steady as the eccentricity is increased in the negative direction (rotor down). Therefore, this fault frequency component is also not suitable for detecting eccentricity fault on the test motor.
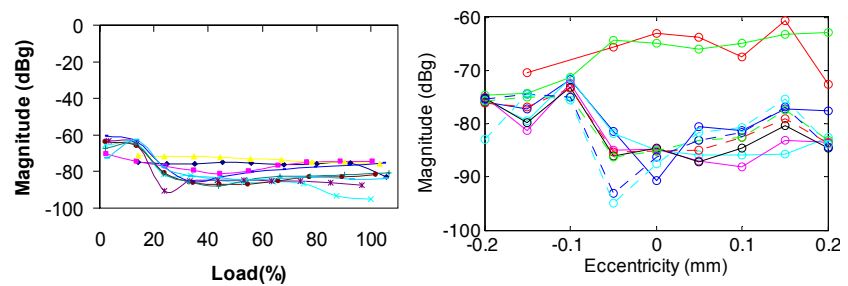

Fig. 9. Magnitude of the component, detected by (7) in the vibration signal, as a function of load (left) and degree of eccentricity (right).
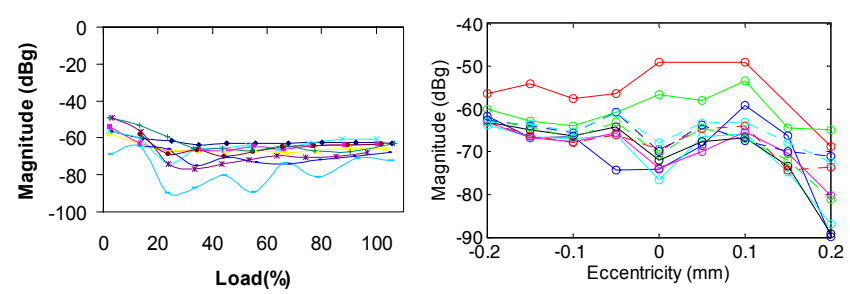

Fig. 10. Magnitude of the component using (8) in the vibration signal, as a function of load (left) and degree of eccentricity (right).

As presented above, it can be concluded from the test results that the eccentricity fault frequency components are not ideal under all loading conditions. Some components are better in eccentricity detection at certain loading conditions, while other components are better at other loads.

\section{UTILIZATION OF FEATURES}

Table II displays the best features that may be utilized in detecting eccentricity faults under the various loading conditions tested. However, the table does not provide any indication about the severity of eccentricity.

It can also be concluded that there are other features that may be useful to distinguish healthy and faulty motors but they are not ideal, where they cannot differentiate the different levels of eccentricity. This paper will identify these features, which are the components of (1-8), by using abbreviations, such as (1a) for the component found in (1) with $n_{d}=1, v=1$ from the current signal. The other abbreviations can be found in Table II. These features include:

- fault frequency component of (1e) at 70-100\% loads and fault frequency component of (2a) at $60-100 \%$ loads, which may be used to separate the different levels of eccentricity but the magnitude of the component is not centred around the concentric rotor position (for the above load ranges, the lowest magnitude is observed at $+12.5 \%$ and $-12.5 \%$ eccentricity respectively).

- fault frequency component of (2b), which can be used to distinguish healthy and faulty motors but it cannot separate the different levels of eccentricity.

- fault frequency component of (2c) at $20-50 \%$ and 60 $70 \%$ loads, which may be used to separate the different levels of eccentricity but the magnitude of the component is not centred around the concentric rotor position (the lowest magnitude is observed at $+12.5 \%$ eccentricity).

- fault frequency component of (7) at 80-100\% loads, which can be used to distinguish healthy and faulty motors without separating the eccentricity levels.

TABLE II

ECCENTRICITY FEATURE SELECTIONS

\begin{tabular}{|l|l|l|}
\hline $\begin{array}{l}\text { Loads } \\
(\%)\end{array}$ & $\begin{array}{c}\text { Good } \\
\text { Eccenctricity } \\
\text { Features }\end{array}$ & \\
\hline $0-10$ & & $1 \mathrm{a} \& 1 \mathrm{~b}=(1)$ with $n_{d}=1, v=+1$ from current and flux signals \\
$10-20$ & $1 \mathrm{f}$ & $1 \mathrm{c} \& 1 \mathrm{~d}=(1)$ with $n_{d}=1, v=-1$ from current and flux signals \\
$20-30$ & $1 \mathrm{f}, 4$ & $1 \mathrm{e} \& 1 \mathrm{f}=(1)$ with $n_{d}=0, v=+1$ from current and flux signals \\
$30-40$ & $1 \mathrm{a}, 1 \mathrm{f}, 2 \mathrm{a}, 4$ & $1 \mathrm{~g} \& 1 \mathrm{~h}=(1)$ with $n_{d}=0, v=-1$ from current and flux signals \\
$40-50$ & $1 \mathrm{a}, 1 \mathrm{f}, 2 \mathrm{a}, 4$ & $2 \mathrm{a} \& 2 \mathrm{~b}=f+f_{r}$ from current and flux signals \\
$50-60$ & $1 \mathrm{f}, 4$ & $2 \mathrm{c} \& 2 \mathrm{~d}=f-f_{r}$ from current and flux signals \\
$60-70$ & $1 \mathrm{f}, 4$ & \\
$70-80$ & $1 \mathrm{f}, 1 \mathrm{~h}, 4$ & \\
$80-90$ & $1 \mathrm{f}, 1 \mathrm{~h}, 4$ & \\
$90-100$ & $1 \mathrm{a}, 1 \mathrm{f}, 1 \mathrm{~h}, 4$ & \\
\hline
\end{tabular}

Although the features selected in Table II is not perfectly ideal (i.e. they can't separate all the eccentricity cases), they can be used to detect and separate most of the different levels of eccentricity. In order to improve the performance of the features, this paper combines all the features in Table II by means of weighted linear combination (WLC), except feature (4) that is not a unique eccentricity feature.

In this method, the weights of each feature determine whether the particular feature is active or inactive. For example, at $30-40 \%$ loads, the features to be activated are (1a), (1f), and (2a) (Table II). Therefore, under this load range the weights of these features are set to 1 (active) while the weights of the other features are set to 0 (inactive). The weighted combination of the eccentricity feature is given by

$$
C(l)=\frac{1}{a(l)} \cdot\left(\widetilde{w}(l) \times \tilde{f}_{t}^{t}\right),
$$

where

$$
\begin{aligned}
& \tilde{w}(l)=\left[\begin{array}{lllll}
w_{1}(l) & w_{2}(l) & w_{3}(l) & w_{1}(l) & \ldots
\end{array}\right], \\
& \widetilde{f}_{t}^{t}=\left[\begin{array}{lllll}
f_{t}(1) & f_{t}(2) & f_{t}(3) & f_{t}(4) & \ldots .
\end{array}\right]^{t},
\end{aligned}
$$

$a$ is the number of features selected for that particular load, $w$ is the weight of each feature, $f_{t}$ is the feature, and $l$ is the loading conditions.

Fig. 11 displays the results of combining multiple eccentricity features (as given in Table II) by means of WLC under various loading conditions and different eccentricity levels.

Combining the eccentricity features through the algorithm in (12) can provide a slightly better ability to separate the different levels of eccentricity in some cases. For example, at $100 \%$ load, the combined magnitude variation, which consists of 3 different features, between the different levels of eccentricity is nearly ideal, where it can distinguish the 
different levels of eccentricity. Each of the features selected contribute to the overall eccentricity WLC and hence should be an improvement over a single feature result. In some other loading conditions, however, the improvements are small because the features selected in Table II are few. It can be noted here that if more good features are obtained, the algorithm (12) should work more effectively and the overall eccentricity fault detection could be improved. The algorithm (12) is also suitable in an automated condition monitoring system because it can extract and combine the useful features based on the loading conditions.
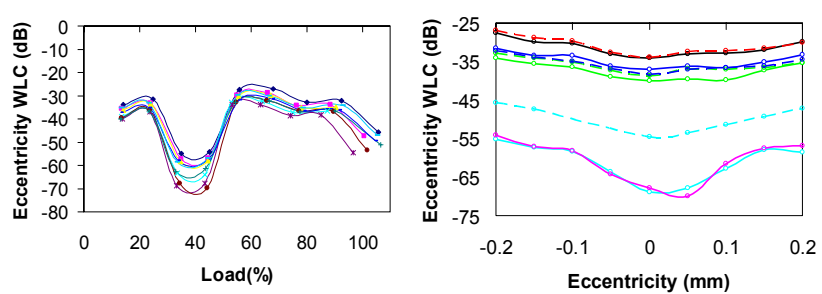

Fig. 11. WLC of eccentricity features as a function of load (left) and eccentricity (right).

It can be emphasised here that it is common to have a misalignment fault when a static eccentricity fault occurs. This is due to the fact that a static eccentricity fault changes the rotor position with respect to the stator airgap and hence the position of the shaft to the coupling (i.e. displacement misalignment), assuming that the motor was originally aligned.

It was found in this study that the eccentricity fault frequency components tested are generally not affected by a misalignment fault and they have similar characteristics as described in the test results, except for components at $3 f_{r}$ (Eqn. 7). The rotor frequency, $f_{r}$, and its harmonics is expected to be susceptible to both eccentricity and misalignment faults.
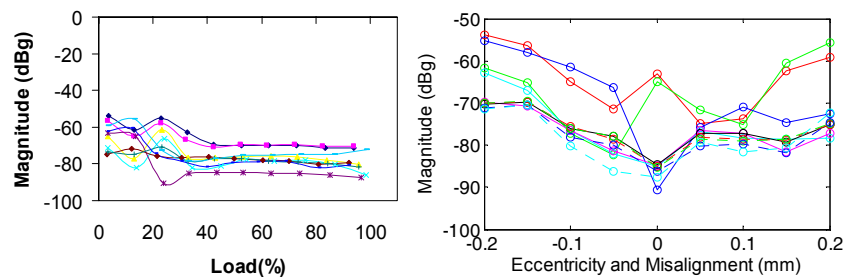

Fig. 12. Magnitude of the peaks detected as a function of load (left) and degree of eccentricity and misalignment (right) by equation (7).

Comparing the results from Figs. 9 and 12, it was found that (7) is affected by misalignment faults but it is less susceptible to eccentricity faults. Figure 9 (right) shows that the magnitude variation in the presence of an eccentricity fault is negligible, while Fig. 12 (right) shows that there is a significant magnitude variation in the presence of combined eccentricity and misalignment faults. These results show that the magnitude variation observed in Fig. 12 can only be caused by the misalignment fault. As a general rule, the greater the severity of the misalignment fault, the greater the magnitude of the fault frequency component. However, the magnitude variation shown in Fig. 12 (right) is not ideal, where it cannot separate some of the misalignment levels.

\section{CONCLUSIONS}

This paper examined the use of fault frequency components in the current, flux and vibration sensor signals with the aim of both detecting and estimating the severity of static eccentricity faults in induction machines in the presence of load variations. Although the method proposed here utilises multiple sensors for eccentricity detection, the cost of such sensors is negligible considering the total cost of an on-line monitoring system. The key results are:

- fault frequency components were identified whose magnitudes increased with increasing levels of eccentricity, however these components were also sensitive to the level of loading;

- the rotor slot passing frequency harmonics (Eqn. 1) and the rotor frequency sidebands of the fundamental (Eqn. 2) in the current and flux signals, and the RMS total vibration signal gave the best results;

- the features obtained from the flux signal seems to be as reliable as, if not better than, the current counterparts.

- it was shown that combining information from multiple frequency components, and taking into account the loading condition can improve the ability to detect and estimate the severity of static eccentricity faults, though even doing this only yielded limited accuracy.

- it was shown that the frequency components for detecting misalignment fault and the components for detecting eccentricity fault do not interfere with each other.

Areas for future research will include investigation of static eccentricity faults on other motor sizes and the use of variable weights in the WLC of eccentricity features.

\section{REFERENCES}

[1] M. L. Sin, W. L. Soong, and N. Ertugrul, "Induction Machine On-Line Condition Monitoring and Fault Diagnosis - A Survey," in Australasian Universities Power Engineering Conference, AUPEC2003, Christchurch, New Zealand, Oct. 2003.

[2] D. G. Dorrell, W. T. Thomson, and S. Roach, "Analysis of Airgap Flux, Current, and Vibration Signals as a Function of the Combination of Static and Dynamic Airgap Eccentricity in 3-Phase Induction Motors," IEEE Transactions on Industry Applications, vol. 33, no. 1, pp. 24-34, Jan.-Feb. 1997.

[3] S. Nandi and H. A. Toliyat, "Condition Monitoring and Fault Diagnosis of Electrical Machines - A Review," in IEEE IAS Annual Meeting, vol. 1, pp. 197-204, Oct. 1999.

[4] S. Nandi, S. Ahmed, and H. A. Toliyat, "Detection of Rotor Slot and Other Eccentricity Related Harmonics in a Three Phase Induction Motor with Different Rotor Cages," IEEE Transactions on Energy Conversion, vol. 16, no. 3, pp. 253260, Sept. 2001.

[5] R. R. Schoen and T. G. Habetler, "Effects of Time-Varying Loads on Rotor Fault Detection in Induction Machines," IEEE Transactions on Industry Applications, vol. 31, no. 4, pp. 900906, July-Aug. 1995.

[6] W. T. Thomson, D. Rankin, and D. G. Dorrell, "On-Line Current Monitoring To Diagnose Airgap Eccentricity In Large Three-Phase Induction Motors - Industrial Case Histories Verify The Predictions," IEEE Transactions on Energy Conversion, vol. 14, no. 4, pp. 1372-1378, Dec. 1999. 